\title{
Avaliação da representatividade do parâmetro de dispersão vertical no cálculo da concentração de um contaminante integrada lateralmente
}

\author{
Andreão, W. L. ${ }^{*}$; Galvão, E. S. ${ }^{1}$; Feroni, R. C. ${ }^{1,2}$ \\ 1 Programa de Pós-Graduação em Engenharia Ambiental, Universidade Federal do Espírito Santo, Vitória, ES, Brasil \\ 2 Centro Integrado de Manufatura e Tecnologia, SENAI-CIMATEC, Salvador, BA, Brasil \\ * e-mail:w.andreao@outlook.com
}

\begin{abstract}
Resumo
A concentração de um contaminante presente no ar pode ser descrita pela equação de difusãoadvecção e utiliza um parâmetro de dispersão vertical que representa o grau de dispersão da pluma no ambiente, que precisa ser estimado. Este trabalho apresenta um comparativo entre as concentrações estimadas a partir dos coeficientes de dispersão vertical em formulação integral, solucionada numericamente via quadratura de Gauss-Legendre, e de formulação algébrica, ambos descritos na literatura. Para as duas formulações também foram testados dois valores para a dissipação de energia média, um sendo uma função dependente da altura da CLC e da estabilidade atmosférica e outro um valor reportado na literatura. A partir da parametrização integral foram estimadas as concentrações de poluentes por meio da equação da pluma Gaussiana integrada lateralmente, concentrações essas que foram confrontadas com os dados experimentais de Copenhagen. Todos os resultados foram analisados estatisticamente para validação do modelo. Os melhores resultados obtidos foram aqueles onde se utilizou uma formulação integral para representar o coeficiente de dispersão vertical e utilizando o valor de 0,65 para a dissipação de energia média.
\end{abstract}

\begin{abstract}
The concentration of a pollutant in the air can be described by the advection-diffusion equation, which uses a vertical dispersion parameter that represents the dispersion of a plume in the ambient and it needs to be estimated. This paper presents a comparison between the concentrations estimated from the vertical dispersion coefficients in integral formulation, solved numerically via Gauss-Legendre quadrature, and from an algebraic formulation, both described in the literature. For the two formulations, it was also tested two values to the average dissipation rate, one being function of $\mathrm{CBL}$ height and atmospheric stability and the other a value reported in the literature. From the integral parameterization, pollutants concentrations were estimated using the equation of laterally integrated Gaussian plume, which were confronted with experimental data of Copenhagen. All results were statistically analyzed to validate the model. The best results were those where an integral formulation was used to represent the vertical dispersion coefficient, and using the value of 0.65 for the average rate dissipation.
\end{abstract}

Keywords (Palavras chaves): Turbulence parameterization (parametrização da turbulência), Convective Boundary Layer (Camada Limite Convectiva), Atmospheric dispersion (Dispersão atmosférica).

\section{Introdução}

A modelagem da dispersão atmosférica se refere à descrição matemática do transporte de contaminantes na atmosfera. A concentração de um contaminante presente no ar pode ser, dessa forma, descrita pela equação de difusão-advecção que é uma equação diferencial parcial de segunda ordem do tipo parabólica. O modelo de pluma gaussiana emprega um sistema de eixos tridimensionais: um a favor do vento, um com vento lateral e outro vertical, com a origem no chão. Também é assumido que as 
concentrações de uma pluma são proporcionais à taxa de emissão e são diluídas pelo vento no ponto de emissão a uma taxa inversamente proporcional à velocidade do vento e que as concentrações médias da dispersão provocada pelo vento lateral e vertical perto da fonte são descritas pela distribuição normal de Gauss. Os desvios-padrões de concentração da pluma nestas duas direções estão empiricamente relacionados com os níveis de turbulência na atmosfera e com o aumento da distância da fonte.

\section{Materiais e Métodos}

O modelo gaussiano que fornece a concentração de um contaminante, integrada lateralmente no nível do solo, e que permite calcular a concentração do contaminante na linha central da pluma no nível do solo é apresentado na Equação 1:

$\bar{C}^{Y}\left(x_{s} z\right)=\frac{Q}{\sqrt{2 \pi} \sigma_{x} U}\left[\exp \left(-\frac{\left(z-H_{2}\right)^{2}}{2 \sigma_{z}^{2}}\right)+\exp \left(-\frac{\left(z+H_{2}\right)^{2}}{2 \sigma_{z}^{2}}\right)\right]$

onde $\overline{c^{Y}}$ é a concentração integrada lateralmente; $H_{g}$ é a altura da fonte; $\sigma_{z}$ a dispersão vertical da pluma (parametrização); $z$ é a altura acima do solo e $U$ é a velocidade média do vento.

A principal dificuldade na aplicação de um modelo Gaussiano é explicar a não-homogeneidade vertical da turbulência nos parâmetros de dispersão. A confiabilidade do modelo, portanto, depende fortemente da forma como os parâmetros de dispersão são calculados e de sua capacidade de reproduzir os resultados de dados experimentais [1].

\subsection{Parametrização 1}

A primeira formulação para o parâmetro de dispersão vertical, descrita pela Equação 2, foi usada para calcular as concentrações de poluentes ao nível do solo a partir de fontes passivas por [1].

$\frac{\sigma_{z}^{2}}{z_{i}^{2}}=\frac{0,093}{\pi} \int_{0}^{\infty} \frac{\sin ^{2}\left(2,96 \psi^{1 / 8} X n v\right)}{(1+n)^{5 / 8}} \frac{d n v}{n t^{2}}$

onde $\sigma_{z}$ é o coeficiente de dispersão vertical; $\psi$ é a dissipação de energia média; $X$ é a distância adimensional (Equação 3); $z_{i}$ é a altura da CLC (m); $n^{\prime}$ é a frequência dos turbilhões.

$X=x w_{*} / U Z_{i}$

onde $x$ é a distância longitudinal da fonte $(\mathrm{m})$; $w_{*}$ é a escala de velocidade convectiva $\left(\mathrm{m} \mathrm{s}^{-1}\right)$.

\subsection{Parametrização 2}

Outro coeficiente de difusão vertical em forma integral utilizado é o encontrado em [2], conforme a Equação 4:

$\frac{\sigma_{z}^{z}}{z_{i}^{z}}=\frac{0,29}{\pi^{2}} \int_{0}^{\infty} \frac{\sin ^{2}\left(0,98 \pi \psi^{1 / s} X n t\right)}{\left(1+n^{J}\right)^{5 / s}} \frac{d n^{J}}{n^{t^{2}}}$

As formas integrais resultantes dos parâmetros de dispersão são mais complicadas do que as algébricas disponíveis na literatura. Por outro lado, elas são mais genéricas, pois, ao contrário das parametrizações algébricas, essas não utilizam medições de dispersão de turbulência, mas, dizem respeito a dispersão da pluma em uma camada instável direta e explicitamente aos tamanhos efetivos dos turbilhões atuando sobre a camada [3].

\subsection{Parametrização 3}

A primeira equação algébrica estudada para 0 coeficiente de dispersão vertical é dada pela Equação 5 e pode ser encontrada em [2].

$\frac{\sigma_{z}^{2}}{z_{i}^{2}}=\frac{0,42 \psi^{2 / s} X^{2}}{1+\left(2,94 \psi^{1 / s} X\right)}$

\subsection{Parametrização 4}

Uma vez que a camada limite convectiva é muito intensa, supõe-se que os desvios padrões da velocidade vertical sejam iguais aos desvios padrões de velocidade lateral $\left(\sigma_{y}=\sigma_{z}\right)$ [4]. Como consequência, o parâmetro de dispersão vertical pode ser expresso pela Equação 6.

$\frac{\sigma_{z}}{z_{\tilde{i}}}=\frac{0,6 X}{(1+2 X)^{1 / 2}}$

\subsection{Dissipação da Energia Média}

A função $\psi$ é a dissipação de energia média e é importante para quantificar parâmetros de dispersão desde que a ordem de magnitude de $\psi$ seja determinada somente pelas quantidades que 
caracterizam os turbilhões mais energéticos [1]. Para o seu cálculo pode-se utilizar a Equação 7.

$\psi^{1 / a}=\left[\left(1-\frac{z}{z_{1}}\right)^{2}\left(\frac{z}{-L}\right)^{-2 / a}+0,75\right]^{1 / 2}$

onde $L$ é o comprimento de Monin-Obukov (m).

Observações de campo da CLC mostram que $\psi$ pode assumir o valor de 0,65, aproximadamente [5]. Dessa forma, os dois valores de $\psi$ (o calculado pela Equação 7 e o dado por [5]) foram testados para cada parametrização do coeficiente de dispersão vertical.

A integral da Equação 2 e da Equação 4 foi solucionada numericamente pela atribuição do método de quadratura de Gauss-Legendre, a partir de um algoritmo computacional em linguagem Matlab baseado na atribuição de pesos e raízes conforme apresentado em [6].

Os resultados das simulações obtidas pelos modelos foram confrontados com dados de concentração observados nos clássicos experimentos realizados na cidade de Copenhagen [7, 8].

\subsection{Experimento de Copenhagen}

Os experimentos de dispersão de Copenhagen foram realizadas sob condições atmosféricas neutras e instáveis. O marcador $\left(\mathrm{SF}_{6}\right)$ foi libertado sem empuxo e a uma taxa constante de uma torre a uma altura de 115 $\mathrm{m}$, e recolhidos em pontos posicionados de 1,9 a 6,1 $\mathrm{km}$ do ponto de lançamento e em unidades de amostragem montadas em postes a uma altura de 2 a 3 metros acima do solo. O local era residencial tendo um comprimento de rugosidade de 0,6 m.

As medições meteorológicas realizadas durante as experiências incluíram flutuações tridimensionais da velocidade e direção do vento e da temperatura na altura de lançamento. A altura de mistura foi determinada por lançamentos diários de radiossondas, realizadas em torno do tempo de amostragem. No conjunto de dados, os valores do comprimento de Monin-Obukov $(L)$ e velocidade de fricção $\left(u_{*}\right)$ foram determinados usando um pré-processador. A Tabela 1 resume os principais parâmetros micrometeorológicos para nove experimentos de dispersão em Copenhagen.

Tabela 1: Parâmetros micrometeorológicos durante o experimento de Copenhagen.

\begin{tabular}{cccccc}
\hline Exp. & $\begin{array}{c}\boldsymbol{U} \\
\left(\mathbf{m ~ s}^{-1}\right)\end{array}$ & $\begin{array}{c}\boldsymbol{u}_{\boldsymbol{*}} \\
\left(\mathbf{m ~ s}^{-1}\right)\end{array}$ & $\begin{array}{c}\boldsymbol{L} \\
(\mathbf{m})\end{array}$ & $\begin{array}{c}\boldsymbol{w}_{\boldsymbol{*}} \\
\left(\mathbf{m ~ s}^{-1}\right)\end{array}$ & $\begin{array}{c}\boldsymbol{z}_{\mathbf{i}} \\
(\mathbf{m})\end{array}$ \\
\hline 1 & 3,4 & 0,36 & -37 & 1,8 & 1980 \\
\hline 2 & 10,6 & 0,73 & -292 & 1,8 & 1920 \\
\hline 3 & 5,0 & 0,38 & -71 & 1,3 & 1120 \\
\hline 4 & 4,6 & 0,38 & -133 & 0,7 & 390 \\
\hline 5 & 6,7 & 0,45 & -444 & 0,7 & 820 \\
\hline 6 & 13,2 & 1,05 & -432 & 2,0 & 1300 \\
\hline 7 & 7,6 & 0,64 & -104 & 2,2 & 1850 \\
\hline 8 & 9,4 & 0,69 & -56 & 2,2 & 810 \\
\hline 9 & 10,5 & 0,75 & -289 & 1,9 & 2090 \\
\hline
\end{tabular}

\section{Resultados}

As Figuras 1 a 7 apresentam 0 diagrama de espalhamento para os valores de concentração integrada lateralmente obtidos pelas parametrizações propostas. A linha central no gráfico representa as concentrações medidas durante os experimentos de Copenhagen e estão normalizadas pela taxa de emissão.

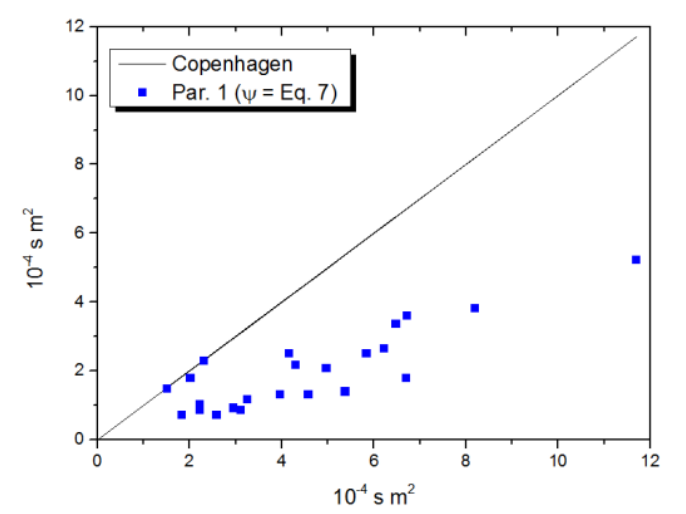

Figura 1: Diagrama de espalhamento para $\sigma_{z}$ dado pela Equação 2 e $\Psi^{*}$ pela Equação 7 .

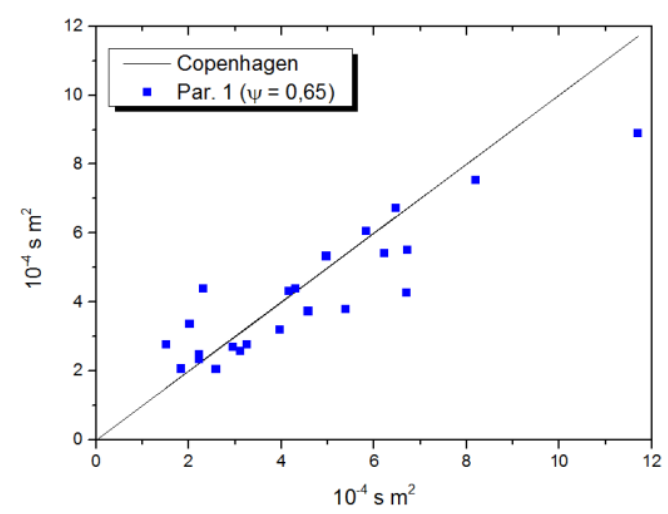

Figura 2: Diagrama de espalhamento para $\sigma_{x}$ dado pela Equação 2 e $\psi^{*}$ $=0,65$. 


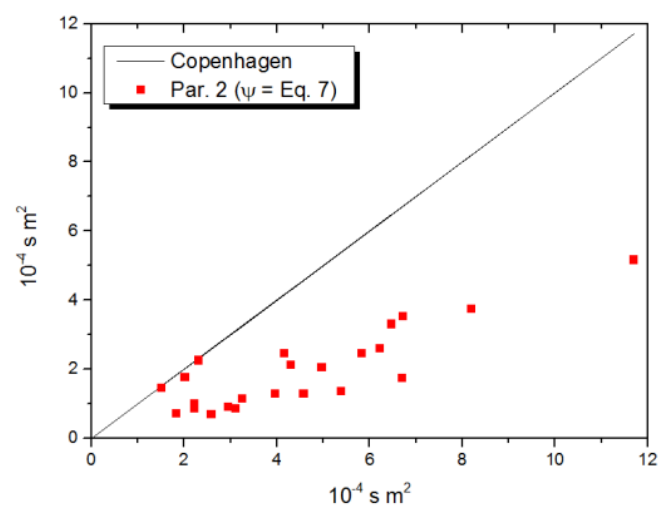

Figura 3: Diagrama de espalhamento para $\sigma_{x}$ dado pela Equação 4 e $\psi$ : pela Equação 7.

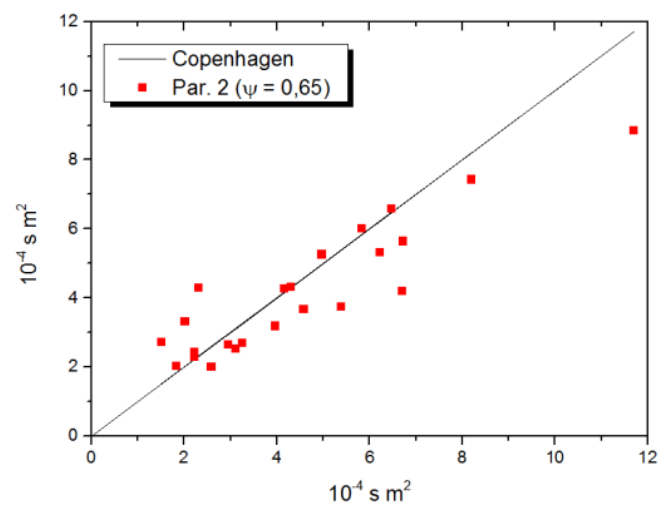

Figura 4: Diagrama de espalhamento para $\sigma_{x}$ dado pela Equação 4 e $\psi^{*}$ $=0,65$.

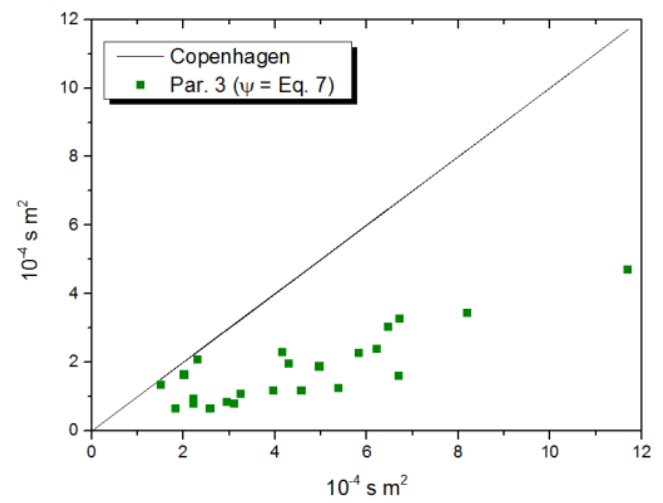

Figura 5: Diagrama de espalhamento para $\sigma_{x}$ dado pela Equação 5 e $\psi$ : pela Equação 7.

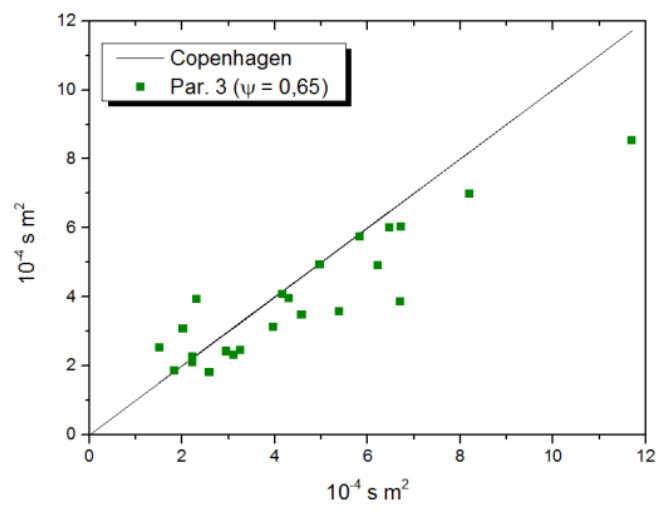

Figura 6: Diagrama de espalhamento para $\sigma_{x}$ dado pela Equação 5 e $=0,65$.

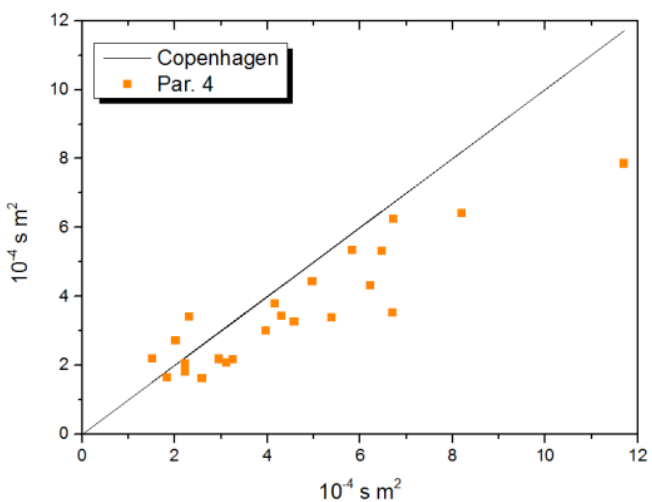

Figura 7: Diagrama de espalhamento para $\sigma_{x}$ dado pela Equação 6

A Tabela 2 apresenta a análise estatística comparativa entre as parametrizações propostas e os dados de Copenhagen.

Tabela 2: Análise estatística comparativa entre as parametrizações e os dados de Copenhagen.

\begin{tabular}{cccccc}
\hline Modelo & Nmse & Cor & Fa2 & Fb & Fs \\
\hline $\begin{array}{c}\text { Par. 1 e } \\
\text { Eq. 7 }\end{array}$ & 0,98 & 0,856 & 0,304 & 0,777 & 0,716 \\
\hline $\begin{array}{c}\text { Par. 1 e } \\
\psi=0,65\end{array}$ & 0,07 & 0,904 & 1,000 & 0,066 & 0,286 \\
\hline $\begin{array}{c}\text { Par. 2 e } \\
\psi \text { Eq. 7 }\end{array}$ & 1,02 & 0,856 & 0,261 & 0,792 & 0,730 \\
\hline $\begin{array}{c}\text { Par. 2 e } \\
\psi=0,65\end{array}$ & 0,07 & 0,907 & 1,000 & 0,079 & 0,291 \\
\hline $\begin{array}{l}\text { Par. 3 e } \\
\psi \text { Eq. 7 }\end{array}$ & 1,24 & 0,853 & 0,174 & 0,862 & 0,805 \\
\hline $\begin{array}{l}\text { Par. 3 e } \\
\psi=0,65\end{array}$ & 0,08 & 0,913 & 1,000 & 0,137 & 0,314 \\
\hline Par. 4 & 0,13 & 0,915 & 1,000 & 0,228 & 0,364 \\
\hline
\end{tabular}

O erro quadrático médio normalizado (Nmse) é a média quadrática normalizada da diferença entre os valores das propriedades observadas e simuladas e apresenta valores de 0 a infinito, tendo como valores ideais, os menores erros e próximos a zero. Todas as parametrizações apresentaram valores satisfatórios, onde a primeira parametrização com $\psi=0,65$ alcançou o menor valor dentre todos $(0,07)$.

O coeficiente de correlação $(r)$ mede a qualidade do ajuste à verdadeira reta, ou seja, a intensidade da relação linear existente entre as variáveis observadas e simuladas. Seu valor pode variar de $-1 \mathrm{a}+1$. Quando não houver relação linear entre as variáveis, o valor de r será zero. Para todas as parametrizações testadas, 
obteve-se um valor de correlação superior a $85 \%$, sendo que a parametrização 4 foi aquela que obteve 0 maior valor $(91,5 \%)$.

O fator de 2 (Fa2) mede a fração de dados (\%) que estão entre $0.5 \leq\left(C_{p} / C_{o}\right) \leq 2$, sendo $C_{p}$ a variável prevista e $\mathrm{C}_{\circ}$ a observada. Todas as parametrizações com $\psi=0,65$ e a parametrização 4 resultaram $100 \%$ dos dados $\left(\mathrm{C}_{\mathrm{p}} / \mathrm{C}_{\mathrm{o}}\right)$ dentro do intervalo 0.5 e 2 . As demais obtiveram valores muito aquém do ideal $(30,4 \%, 26,1 \%$ e $17,4 \%$ para as parametrizações 1,2 e 3 respectivamente).

Valores negativos de erro fracional (fractional bias FB) mostram que o modelo está superestimando a média dos valores previstos e para valores positivos de FB, o modelo subestima tal média. O valor ótimo esperado para este teste é zero. Os maiores valores assumidos por este erro são - 2 e 2 . Em todas as parametrizações testadas o erro é positivo, o que mostra que os valores médios estão subestimados. A parametrização $1 \mathrm{com} \psi=0,65$ obteve o melhor resultado $(0,066)$.

O desvio padrão fracional (fractional standard - FS), confronta as variáveis simuladas com as observadas, mostrando se a variável média simulada está superestimada ou subestimada em torno da variável média. $O$ valor ótimo para esse desvio também é zero. Os valores de FS podem variar de - 2 a 2 . Mais uma vez, as parametrizações que utilizaram $\psi=0,65$ obtiveram os melhores resultados. A parametrização 4 também obteve um valor satisfatório. A parametrização 1 com $\psi=0,65$ apresentou o melhor resultado $(0,286)$.

Com base no contexto acima, pode-se afirmar que a parametrização $1 \mathrm{com} \psi=0,65$ foi a que melhor representou os dados observados do experimento de Copenhagen.

\section{Conclusão}

Os resultados apresentados neste trabalho indicam que a parametrização do coeficiente de dispersão vertical apresentado pela Equação 2 representa satisfatoriamente os fenômenos turbulentos e de dispersão vertical, principalmente quando o valor de 0,65 é usado para descrever a dissipação de energia média.

A solução numérica das equações do coeficiente de dispersão vertical pelo método de quadratura de Gauss-Legendre se mostrou satisfatória. As formas integrais para o cálculo do $\sigma_{z}$ são mais genéricas e podem melhor representar os turbilhões atuando sobre a CLC [3]. De fato, a parametrização $1 \mathrm{com} \psi=0,65$ foi a que apresentou os melhores resultados.

\section{Referências}

[1] MOREIRA, D. M.; SANTOS, D. P.; CARVALHO, J. C.; VILHENA, M. T.; DEGRAZIA, G. M.; TIRABASSI, T. A. Revista Brasileira de Meteorologia, v. 19, p. 113-122, 2004.

[2] BULIGON, L.; DEGRAZIA, G. A.; SZINVELSKIA, C. R. P.; GOULARTB, A. G. The Open Atmospheric Science Journal, v. 2, p. 153-159, 2008.

[3] MANGIA, C.; MOREIRA, D. M.; SCHIPA, I.; DEGRAZIA, G.A.; TIRABASSI, T.; RIZZA, U. Atmospheric Environmental, v. 36, p. 67-76, 2002.

[4] SASTRE, F. A. U.; CAMPOS, C. R. J. de; DEGRAZIA, G. A.; MOREIRA, D. M. Revista Brasileira de Meteorologia, v. 20, p. 165-174, 2005.

[5] CAUCHEY, S. J.; PALMER, S. G. Quarterly Journal of the Royal Meteorological Society, v. 105, p. 811-827, 1979.

[6] Introdução ao Matlab, Belo Horizonte. Disponível em <http://www.ime.unicamp.br/ encpos/VIII_EnCPos/Apo stila_Matlab.pdf>. Acesso em: 30 Abr. 2016.

[7] GRYNING, S. E.; LYCK E. Journal of Applied Meteorology and Climatology, v. 23, p. 651-660, 1984.

[8] GRYNING, S.-E.; HOLTSLAG, A. A. M.; IRWIN, J. S.; SIVERTSEN, B. Atmospheric Environmental, v. 21, p. 79-89, 1987. 